\title{
Costes de los agentes estimulantes de la eritropoyesis en el tratamiento de la anemia inducida por quimioterapia en España: resultados preliminares procedentes de la adaptación de un estudio belga
}

\author{
Aurea Duran • Erik Spaepen • Mark Lamotte • \\ Max Brosa - Laura Gutiérrez • Gabriela Restovic \\ Published online: 26 September 2013 \\ (C) The Author(s) 2013. This article is published with open access at Springerlink.com
}

Resumen Objetivos Spaepen y cols. (The Oncologist 13: 596-607, 2008) compararon el coste de manejo de la anemia inducida por quimioterapia en pacientes tratados con darbepoetina-alfa (DARB), epoetina-alfa (EPO-A) y epoetinabeta (EPO-B) en Bélgica. Los objetivos del presente estudio fueron determinar la aplicabilidad del estudio belga en España y evaluar las diferencias de coste entre los agentes estimulantes de la eritropoyesis (AEE) en dicho país.

Métodos Las diferencias en la epidemiología del cáncer y los patrones de tratamiento entre Bélgica y España se ajustaron usando datos de Eurostat, registros nacionales de cáncer, ventas de IMS y guías de tratamiento. Se usaron los costes unitarios españoles para sustituir los costes belgas. Los costes totales se estimaron usando un modelo de efectos-mixtos estratificando por índice de propensión en quintiles.

Resultados Las dos poblaciones fueron similares en edad; sexo; y uso de AEE, hierro y transfusiones sanguíneas. Tras

\author{
A. Duran \\ Health Economics and Outcomes Research, IMS Health, \\ 210 Pentonville Road, Londres, N1 9JY, Reino Unido \\ e-mail: aurea.duran@uk.imshealth.com \\ E. Spaepen \\ SBD Analytics BVBA/SPRL, Bekkevoort, Bélgica \\ M. Lamotte $(\varangle)$ \\ Medialaan 38, 1800 Vilvoorde, Bélgica \\ e-mail: MLamotte@be.imshealth.com \\ M. Brosa \\ Oblikue Consulting, Barcelona, España \\ L. Gutiérrez \\ Amgen S.A., Barcelona, España \\ G. Restovic \\ Consultora independiente, Barcelona, España
}

ajustar los datos belgas de quimioterapia e incidencia de cáncer para España, los costes totales por paciente (Euros, 2010) con DARB fueron $6.815 €$, un $21 \%$ inferiores a los de EPO-A (8.583 $€ ; p<0,0001)$ y un $19 \%$ inferiores en comparación con EPO-B (8.420 $€ ; p=0,0019)$. La duración del tratamiento fue de 40,63 $\pm 2,39$ días con DARB, $53,59 \pm 1,25$ días con EPO-A y $52,39 \pm 2,54$ días con EPO-B.

Conclusión En línea con los resultados de Spaepen (2008), los costes totales y los asociados al tratamiento de la anemia fueron más bajos en los pacientes tratados con DARB. Este estudio demuestra la viabilidad de adaptar los datos específicos de un país a otros entornos, ajustando por las diferencias en las características de los pacientes y las estrategias de tratamiento.

Palabras clave Anemia - Cáncer - Quimioterapia · Agentes estimuladores de la eritropoyesis · Costes Abstract Objectives Spaepen et al. (The Oncologist 13:
596-607, 2008) compared the management cost of chemo-
therapy-induced anaemia in patients treated with darbepo-
etin-alfa (DARB), epoetin-alfa (EPO-A), and epoetin-beta
(EPO-B) in Belgium based on a database analysis, and con-
cluded that the cost in patients treated with DARB was
significantly lower than in patients treated with EPO-A or
EPO-B. The objectives of present study were to determine
the applicability of the Belgian analysis to Spain, and to
estimate the differences in costs between erythropoiesis-
stimulating agents (ESAs) in Spain.
Methods Differences in epidemiology and treatment patterns
between Belgium and Spain were adjusted using data from
Eurostat, national cancer registries, IMS sales data, and re-
imbursement and treatment guidelines. Belgian unit costs 
were replaced with Spanish-specific costs. Costs were analyzed using a mixed-effects model stratifying for propensity score quintiles.

Results The two populations were comparable in terms of age; gender; and ESA, iron, and blood transfusions use. After adjusting for Spanish-specific chemotherapy use and cancer incidence, total management costs per patient (Euro, 2010) for DARB were $€ 6,815,21 \%$ lower than for EPO-A $(€ 8,583 ; p<0.0001)$ and $19 \%$ lower than for EPO-B $(€ 8,420 ; p=0.0019)$. The duration of treatment was $40.63 \pm 2.39$ days for DARB, $53.59 \pm 1.25$ days for EPO-A and $52.39 \pm 2.54$ days for EPO-B.

Conclusion The findings of this study are in line with the Belgian analysis: total costs and anaemia-related costs were lowest in patients receiving DARB compared with EPO-A or EPO-B. This study demonstrates the feasibility of adapting real-life country-specific data to other settings, adjusting for differences in patients' characteristics and treatment strategies.

Keywords Anaemia - Cancer - Chemotherapy . Erythropoiesis-stimulating agents $\cdot$ Costs

\section{Introducción}

Diversos estudios han demostrado que tanto la anemia inducida por la quimioterapia (AIQ) como la fatiga resultante tienen un importante impacto negativo en la vida diaria de los pacientes con cáncer [1-4]. Los agentes estimulantes de la eritropoyesis (AEE) se utilizan en el tratamiento de la AIQ para corregir los niveles insuficientes de hemoglobina $(\mathrm{Hb})$, mejorar la calidad de vida y reducir la necesidad de realizar transfusiones sanguíneas [5]. También se han observado mejoras en la fatiga de los pacientes tratados con AEE [6]. Asimismo, los pacientes con niveles de $\mathrm{Hb}$ adecuados pueden recibir el tratamiento de quimioterapia necesario más pronto que los pacientes que continúan estando anémicos [7-9]. Los AEE utilizados para el tratamiento de la anemia en pacientes con cáncer en España son la darbepoetina-alfa (DARB), la epoetina-alfa (EPO-A) y la epoetina-beta (EPO-B).

Dada la notable carga económica asociada al tratamiento de los pacientes con cáncer, es relevante entender los costes relacionados con el tratamiento de la AIQ, así como cualquier posible ahorro que pudiera lograrse usando uno u otro AEE. En 2008, Spaepen y cols. publicaron los resultados de una evaluación económica en la que se compararon los costes y los resultados de la DARB (solamente disponible en el régimen semanal $(\mathrm{QW})$ durante el periodo en que la información fue recogida), la EPO-A y la EPO-B en el tratamiento de la AIQ en Bélgica [10]. Los autores concluyeron que, tras ponderar el peso de los pacientes según el índice de propensión, el coste relacionado con el tratamiento de la anemia fue un 20\% inferior con el uso de DARB en comparación con lo observado al usar EPO-A o EPO-B, lo que podía ser debido a la menor duración del tratamiento con DARB en comparación con EPO-A y EPO-B. Asimismo, para obtener resultados clínicos comparables (tasa de supervivencia libre de transfusiones y reingresos por anemia del $84,37 \%$ con DARB, 84,60\% con EPO-A y 84,94\% con EPO-B), los pacientes tratados con DARB requirieron proporcionalmente menos AEE que los tratados con EPO-A o EPO-B [10].

Dada la naturaleza de los datos (observacionales y retrospectivos), existía un riesgo potencial de sesgo de selección en la asignación, no aleatoria, de los pacientes a los diferentes AEE [11]. Para corregir este sesgo, los investigadores desarrollaron un índice de propensión utilizando 13 variables relacionadas con la epidemiología y los patrones de tratamiento de la AIQ. Este método, comúnmente utilizado en estudios observacionales [12-14], consiste en asignar pacientes a los distintos grupos de tratamiento (estratos) y posteriormente realizar un análisis estratificado convencional. Con ello se consigue simular la asignación aleatoria [15]. Para aplicar esta metodología se debe disponer de una base de datos con información longitudinal y detallada a nivel del paciente.

El análisis realizado en Bélgica se basó en información derivada de la Base de Datos de Enfermedades Hospitalarias de IMS (IMS HDD, del inglés IMS Hospital Disease Database), datos de 2003 a 2005, e incluyó un total de 2.513 pacientes (Tabla 1) [10]. Esta es una base de datos longitudinal que contiene información sobre diagnósticos, procedimientos y productos farmacéuticos a nivel de paciente [10], que incluye información de 46 de los 110 hospitales de Bélgica, y que representa el $34 \%$ de las visitas al hospital de día e ingresos hospitalarios del país. Dado que en Bélgica los AEE solo pueden ser dispensados por la farmacia del hospital, la IMS HDD refleja todo el uso de AEE en pacientes con cáncer. En cuanto al nivel de detalle y exhaustividad de la información, la IMS HDD es comparable a la General Practice Research Database (GPRD) del Reino Unido [16], pero a diferencia de ésta, que se centra en atención primaria, la IMS HDD contiene información sobre la atención hospitalaria.

Los objetivos del presente estudio fueron determinar la aplicabilidad del estudio belga presentado por Spaepen y cols. [10], manteniendo la calidad metodológica derivada del uso de una base de datos retrospectiva completa, al ámbito sanitario español y evaluar las diferencias de coste entre los agentes estimuladores de la eritropoyesis (AEE) en el tratamiento de la anemia inducida por quimioterapia en España.

\section{Métodos y materiales}

Con el fin de estimar las diferencias de costes derivadas del tratamiento de la AIQ con los distintos AEE, se llevó a ca- 
Tabla 1 Análisis general

1 Muestra inicial de pacientes

IMS HDD, enero 2003-junio 2005

Pacientes con cáncer, tratados con quimioterapia y AEE

DARB $(n=539)$; EPO-A $(n=1.594)$, EPO-B $(n=380)$

2 Apareamiento por índice de propensión

DARB $(n=429)$; EPO-A $(n=1.584)$, EPO-B $(n=380)$

3 Costes unitarios españoles

En pacientes apareados por índice de propensión, se sustituyeron los costes belgas por costes españoles:

- Costes de medicamentos (sustitución de los Precios de Venta del Laboratorio)

- Costes de procedimientos (sustitución a nivel de GRD)

- Costes de hospitalización (cálculo del coste por día en base a los GRD en España y multiplicación por la duración de la hospitalización)

4 Ponderación de la muestra

Se ajustó la muestra con los pesos calculados para reflejar el uso de quimioterapia y la incidencia de cáncer en España:

Uso de quimioterapia:

- Razón del número de unidades de quimioterapia usadas en España frente a Bélgica.

- La media ponderada de las unidades de quimioterapia usadas se utiliza como peso en el análisis.

- Una razón más elevada indica un mayor uso de quimioterapia, y mayor peso para el paciente.

Incidencia del cáncer:

- Peso relativo de la incidencia de 4 tipos de cáncer (mama, pulmón, ovario y hematológico) en España en comparación con Bélgica.

- Una razón más elevada indica una incidencia relativa superior, y por tanto mayor peso del paciente.

5 Ponderación final

1. Peso final: ponderación quimioterapia $x$ ponderación incidencia

2. Peso final normalizado: Peso final ajustado por el tamaño muestral por grupos

IMS HDD = IMS Hospital Disease Database; $\mathrm{AEE}=$ agentes estimuladores de la eritropoyesis; DARB = darbepoetina-alfa; EPO-A = epoetinaalfa; $\mathrm{EPO}-\mathrm{B}=$ epoetina-beta; GRD = grupo relacionado por el diagnóstico

bo un proceso de identificación y revisión de bases de datos longitudinales disponibles en España, para determinar si contenían información a nivel individual del paciente sobre consumo de medicamentos, diagnóstico y costes de atención secundaria. Dicha revisión concluyó, en línea con la evidencia publicada en la base de datos gestionada por ISPOR (International Society for Pharmacoeconomics and Outcomes Research) [17], que en España no existía ninguna base de datos longitudinal de características similares a la IMS HDD. Por tanto, se evaluó la posibilidad de replicar en España el análisis llevado a cabo con la base de datos IMS HDD de Bélgica [10], ajustando los perfiles de las 2 poblaciones mediante agrupación de los pacientes según el índice de propensión. Para ello se utilizó el método de estandarización indirecta de Kirkwood y Sterne [18] según el cual las tasas demográficas y epidemiológicas de una población estándar se pueden aplicar a una población objeto de estudio.

Equivalencia entre las poblaciones belga y española

Para poder utilizar el conjunto de datos original, en primer lugar se estableció la equivalencia entre las poblaciones española y belga para aquellos parámetros que representaban un mayor impacto en los resultados del análisis original [10]. En términos demográficos y epidemiológicos, se analizaron las estadísticas demográficas generales de la población española, así como la incidencia y la distribución de los distintos tipos de cáncer. Los patrones de tratamiento analizados incluyeron el uso de AEE, de transfusiones de sangre y de quimioterapia.

\section{Costes unitarios españoles}

Los costes unitarios de los distintos productos farmacéuticos en Bélgica fueron reemplazados por precios de venta del laboratorio (PVL) en España, calculados a partir de la base de datos del Consejo General del Colegio Oficial de Farmacéuticos (CGCOF) [19]. Los PVL de los distintos AEE utilizados en el análisis fueron $249,68 €$ por vial de 150 microgramos $(\mathrm{mcg})$ de DARB $(1,66 € / \mathrm{mcg}), 334,04 €$ por vial de 40.000 unidades internacionales (UI) de EPO-A $(1,67$ $€ / \mathrm{mcg})$ y $83,77 €$ por vial de 10.000 UI de EPO-B $(1,67$ $€ / \mathrm{mcg}$ ). Los costes de los grupos relacionados por el diagnóstico (GRD) incluidos en el análisis belga fueron reemplazados por sus equivalentes en España [20]. Los costes de procedimientos como las transfusiones de sangre y costes de hospitalización se obtuvieron de la base de datos de costes sanitarios eSalud [21]. Todos los costes unitarios se refieren a euros de 2010. 
Fuente de datos demográficos y epidemiológicos

La información demográfica sobre la distribución de la población por edad y sexo se obtuvo de Eurostat, que proporciona datos estadísticos armonizados de todos los Estados miembros de la Unión Europea [22]. La información sobre la incidencia de los distintos tipos de tumores en Bélgica (2006) [23] y España (2008) [24] se obtuvo de fuentes específicas para cada país. Estos datos se clasificaron mediante la décima revisión de la Clasificación Internacional de Enfermedades (CIE-10) [25]. Al comparar estos datos de incidencias se encontraron diferencias significativas entre los datos españoles y belgas en cuatro tipos de tumores (cáncer hematológico, cáncer de mama femenino, cáncer de pulmón y cáncer de ovario). Se utilizó el peso relativo de estos tipos de tumores ajustándolos al conjunto de datos original para reflejar las diferencias entre España y Bélgica en la incidencia de los distintos tipos de cáncer.

\section{Patrones de tratamiento}

Puesto que los patrones de tratamiento de pacientes con cáncer pueden influir tanto en la incidencia de la AIQ como en los costes medios estimados en el análisis, se comparó la información sobre el uso y reembolso de los AEE en ambos países. Al no encontrarse directrices específicas a nivel nacional para el uso de transfusiones de sangre en ningún país, se asumió que tanto Bélgica como España siguen las pautas marcadas por la Organización Europea para la Investigación y Tratamiento del Cáncer (EORTC) [26].

Además, para poder capturar las diferencias entre los regímenes de quimioterapia utilizados en ambos países, se calculó el número de unidades de quimioterapia (miligramos) usadas por paciente. Esta información se obtuvo del Sistema Integrado Multinacional de Análisis de Datos de IMS (MIDAS) Quantum (2009) para Bélgica y España [27]. Para determinar la clase anatómica terapéutica (ATC) correspondiente a los agentes quimioterapéuticos se utilizaron las directrices de clasificación anatómica de productos farmacéuticos (2010) [28]. Dentro de la sección L (Agentes antineoplásicos e inmunomoduladores), se tuvieron en cuenta las subsecciones L01 (antineoplásicos) y L02 (terapia hormonal citostáticos). Para todas las moléculas de las clases L01 y L02 se calcularon los pesos relativos del consumo en España frente a Bélgica en miligramos.

\section{Análisis general}

En primer lugar se ajustó el conjunto de datos belga original para reflejar la utilización de quimioterapia y la incidencia del cáncer en España. Para ello se calculó un peso total normalizado incluyendo la razón de incidencia de cáncer entre España y Bélgica y el consumo medio de la quimioterapia en miligramos por molécula. Este peso fue incluido en el ajuste por el índice de propensión [10], lo que permitió mantener la relación establecida entre los distintos parámetros para conseguir así que los resultados en España fueran comparables con los de Bélgica.

Posteriormente se calcularon los costes sanitarios totales y los relacionados con la anemia para cada uno de los tres AEE, mediante un modelo jerárquico mixto de estratificación por quintiles del índice de propensión, tal y como se describe en Spaepen (2008) [10]. Los costes sanitarios totales de manejo de los pacientes incluían los costes de los productos farmacéuticos (AEE, productos quimioterapéuticos y otros), los costes de los procedimientos (por ejemplo, la administración de quimioterapia o las transfusiones) y los costes de hospitalización de los pacientes (cama hospitalaria con supervisión médica). Los costes relacionados con la anemia incluían los costes de los AEE, las transfusiones de sangre y cualquier otro gasto en el que se incurriera durante la hospitalización de los pacientes como consecuencia de la anemia (por ejemplo, los costes del suplemento de hierro).

En línea con el análisis presentado por Duran y cols. [29], se realizó un análisis de sensibilidad para determinar el impacto de realizar este estudio basado en precios de venta al público (PVP). A fin de comparar el uso de los diferentes AEE para el tratamiento de la AIQ se llevó a cabo un análisis de eficiencia, para el cual el número medio de UI de EPO-A y EPO-B se convirtió a mcg utilizando un factor de conversión de 200 UI $=1 \mathrm{mcg}$. También se realizó un análisis de los días de duración del tratamiento.

\section{Resultados}

Después de ajustar los datos por el índice de propensión, el estudio incluyó un total de 2.393 pacientes (DARB, $n=429$; EPO-A, $n=1.584$; EPO-B, $n=380$ ) (Tabla 1).

El perfil demográfico de las poblaciones de Bélgica y España fue similar en ambos países en términos de edad (media y distribución), sexo e incidencia de cáncer, como se muestra en la Tabla 2.

Se usaron las razones de la incidencia de los 4 tipos de cáncer incluidos en el análisis en España respecto a Bélgica para realizar ajustes en el conjunto de datos original y reflejar así las características específicas de España. La Tabla 3 presenta esas razones.

El porcentaje de pacientes tratados con hierro fue similar en los pacientes que recibieron tratamiento con DARB (6\%), con EPO-A (6\%) y con EPO-B (5,8\%). La dosis media del tratamiento (expresada en microgramos) con DARB fue inferior a la de los otros AEE. La dosis media del tratamiento con DARB fue de $1.020 \mathrm{mcg}$, mientras que con EPOA fue $1.381 \mathrm{mcg}(p<0,001)$ y con EPO-B fue de 1.181 $\operatorname{mcg}(p=0,0575)$. En el análisis del uso de quimioterapia, 
los antineoplásicos (de la clase ATC L01) representaron la mayor parte del coste de los agentes antineoplásicos e inmu-

Tabla 2 Perfiles demográficos y epidemiológicos

\begin{tabular}{llll}
\hline & & Bélgica & España \\
\hline Población total & Mujeres & 5.442 .557 & 22.921 .983 \\
& Hombres & 5.224 .309 & 22.355 .470 \\
& TOTAL & 10.666 .866 & 45.277 .453 \\
\% población & Mujeres & $51,02 \%$ & $50,63 \%$ \\
& Hombres & $48,98 \%$ & $49,37 \%$ \\
Media de edad & TOTAL & $100 \%$ & $100 \%$ \\
& Mujeres & 42,00 & 42,05 \\
Total cáncer & Hombres & 39,39 & 39,38 \\
& TOTAL & 40,72 & 40,73 \\
& Mujeres & 25.166 & 111.297 \\
& Hombres & 30.501 & 160.755 \\
& TOTAL & 55.667 & 272.052 \\
& Mujeres & $0,46 \%$ & $0,49 \%$ \\
& Hombres & $0,58 \%$ & $0,72 \%$ \\
& TOTAL & $0,52 \%$ & $0,60 \%$ \\
\hline
\end{tabular}

Tabla 3 Incidencia relativa del cáncer ${ }^{\mathrm{a}}$

España

Cáncer hematológico

1,2094

Cáncer de mama femenino

0,5654

Cáncer de pulmón

0,6716

Cáncer de ovario

0,9589

${ }^{a}$ Razón de España sobre Bélgica nomoduladores prescritos, tanto en Bélgica $(97,01 \%)$ como en España $(93,93 \%)$, en comparación con la terapia hormonal (ATC L02).

Al igual que en el análisis original llevado a cabo en Bélgica, y después de realizar los ajustes pertinentes para reflejar la incidencia de cáncer y el uso de quimioterapia específicos para España, la duración del tratamiento con DARB fue significativamente inferior a la de los otros AEE. La duración media del tratamiento con DARB se estimó en 40,63 días, mientras que con EPO-A fue de 53,59 días $(p<0,0001)$ y con EPO-B fue de 52,39 días $(p=0,0008)$. Por tanto, el ahorro de días con DARB respecto a EPO-A fue del $24,18 \%$, y respecto a EPO-B del $22,45 \%$. Los resultados se presentan en la Tabla 4. Los ajustes realizados tuvieron un efecto similar en la duración media estimada de hospitalización para pacientes tratados con DARB $(6,58$ días), comparados con los tratados con EPO-A (10,39 días; $p<0,0001)$ o EPO-B (8,05 días; $p=0,2447)$. Del mismo modo, el porcentaje de pacientes que tratados con quimioterapia que fueron hospitalizados fue inferior en el grupo tratado con DARB $(9,2 \%)$, en comparación con el $10,1 \%$ $(p<0,0001)$ y el $10,0 \%(p=0,0051)$ de EPO-A y EPO$\mathrm{B}$, respectivamente. El porcentaje de pacientes tratados con DARB que recibieron transfusiones sanguíneas con DARB $(39,6 \%)$ fue inferior al observado en los pacientes tratados con EPO-A $(44,4 \% ; p=0,0735)$ y mayor que el de los pacientes tratados con EPO-B $(33,9 \% ; p=0,1072)$, aunque ninguna de estas diferencias fue estadísticamente significativa.

Los costes sanitarios totales por paciente tratado con DARB ascendieron a $6.815 €$, mientras que con EPO-A ascendieron a $8.583 €(p<0,0001)$ y con EPO-B a $8.420 €(p=0,0019)$. Los costes relacionados con la

Tabla 4 Uso de recursos necesario en el manejo de pacientes con AIQ en Bélgica y en España

\begin{tabular}{llllll}
\hline & & $\begin{array}{l}\text { Bélgica } \\
(\text { media } \pm \text { DE) }\end{array}$ & $\begin{array}{l}\text { Diferencias respecto } \\
\text { a DARB (media } \pm \text { DE) }\end{array}$ & $\begin{array}{l}\text { España } \\
\text { (media } \pm \text { DE) }\end{array}$ & $\begin{array}{l}\text { Diferencias respecto } \\
\text { a DARB (media } \pm \text { DE) }\end{array}$ \\
\hline Duración del tratamiento (días) & DARB & $41,39 \pm 2,33$ & & $40,63 \pm 2,39$ & \\
& EPO-A & $52,82 \pm 1,28$ & $11,43 \pm 2,57(p<0,0001)$ & $53,59 \pm 1,25$ & $12,96 \pm 2,70(p<0,0001)$ \\
& EPO-B & $53,22 \pm 2,47$ & $11,84 \pm 3,34(p=0,0004)$ & $52,39 \pm 2,54$ & $11,76 \pm 3,49(p=0,0008)$ \\
Duración de la hospitalización & DARB & $6,79 \pm 2,42$ & & $6,58 \pm 2,54$ & \\
(días) & EPO-A & $9,59 \pm 2,33$ & $2,81 \pm 0,89(p=0,0017)$ & $10,39 \pm 2,43$ & $3,81 \pm 0,96(p<0,0001)$ \\
& EPO-B & $9,05 \pm 2,44$ & $2,26 \pm 1,18(p=0,0549)$ & $8,05 \pm 2,56$ & $1,47 \pm 1,26(p=0,2447)$ \\
\% hospitalizaciones & DARB & $9,1 \% \pm 0,2 \%$ & & $9,2 \% \pm 0,2 \%$ & \\
relacionadas con quimioterapia & EPO-A & $10,0 \% \pm 0,2 \%$ & $0,9 \% \pm 0,22 \%(p<0,0001)$ & $10,1 \% \pm 0,1 \%$ & $0,9 \% \pm 0,22 \%(p<0,0001)$ \\
(\% del total) & EPO-B & $10,1 \% \pm 0,2 \%$ & $0,9 \% \pm 0,29 \%(p=0,0009)$ & $10,0 \% \pm 0,2 \%$ & $0,8 \% \pm 0,29 \%(p=0,0051)$ \\
\% pacientes con transfusiones & DARB & $37,5 \% \pm 3,5 \%$ & & $39,6 \% \pm 3,1 \%$ & \\
sanguíneas & EPO-A & $39,9 \% \pm 2,9 \%$ & $2,4 \% \pm 2,63 \%(p=0,3646)$ & $44,4 \% \pm 2,3 \%$ & $4,8 \% \pm 2,67 \%(p=0,0735)$ \\
& EPO-B & $34,7 \% \pm 3,6 \%$ & $-2,8 \% \pm 3,47 \%(p=0,4188)$ & $33,9 \% \pm 3,2 \%$ & $-5,7 \% \pm 3,51 \%(p=0,1072)$ \\
\hline
\end{tabular}

$\mathrm{DE}=$ Desviación estándar; DARB = darbepoetina-alfa; EPO-A = epoetina-alfa; EPO-B = epoetina-beta; AEE = agentes estimuladores de la eritropoyesis 
Tabla 5 Costes sanitarios totales y costes relacionados con la anemia en España

\begin{tabular}{|c|c|c|c|c|c|}
\hline & $\begin{array}{l}\text { DARB } \\
\text { (media) }\end{array}$ & $\begin{array}{l}\text { EPO-A } \\
\text { (media) }\end{array}$ & Diferencias respecto a DARB & $\begin{array}{l}\text { EPO-B } \\
\text { (media) }\end{array}$ & Diferencias respecto a DARB \\
\hline \multicolumn{6}{|c|}{ Caso base: precios de laboratorio } \\
\hline Costes totales & 6.815 & 8.583 & $1.768(p<0,0001)[$ IC 95\% 986; 2524] & 8.420 & $1.605(p=0,0019)$ [IC 95\% 662; 2.684] \\
\hline Costes totales de la anemia & 2.246 & 2.972 & $726(p<0,0001)[$ IC $95 \% 448 ; 1.013]$ & 2.425 & $179(p=0,2531)[$ IC $95 \% 152 ; 578]$ \\
\hline Costes de los AEE & 1.698 & 2.308 & $610(p<0,0001)[$ IC 95\% 395; 824] & 1.958 & $260(p=0,0659)[$ IC 95\% 17; 537] \\
\hline \multicolumn{6}{|c|}{ Análisis de sensibilidad: precios públicos } \\
\hline Costes totales & 7.747 & 10.466 & $2.719(p<0,0001)[$ IC 95\% 1.774; 3.664] & 9.723 & $1.976(p=0,0019)[$ IC $95 \% 731 ; 3.222]$ \\
\hline Costes totales de la anemia & 2.378 & 3.349 & $971(p<0,0001)[$ IC 95\% 654; 1.289] & 2.857 & $479(p=0,0226)[\mathrm{IC} 95 \% 67 ; 890]$ \\
\hline Costes de los AEE & 1.827 & 2.675 & $848(p<0,0001)$ [IC 95\% 600; 1.097] & 2.348 & $521(p=0,0015)[$ IC $95 \% 199 ; 843]$ \\
\hline
\end{tabular}

IC = intervalo de confianza; DARB = darbepoetina-alfa; EPO-A = epoetina-alfa; EPO-B = epoetina-beta; AEE = agentes estimuladores de la eritropoyesis

Figura 1 Costes sanitarios medios por paciente

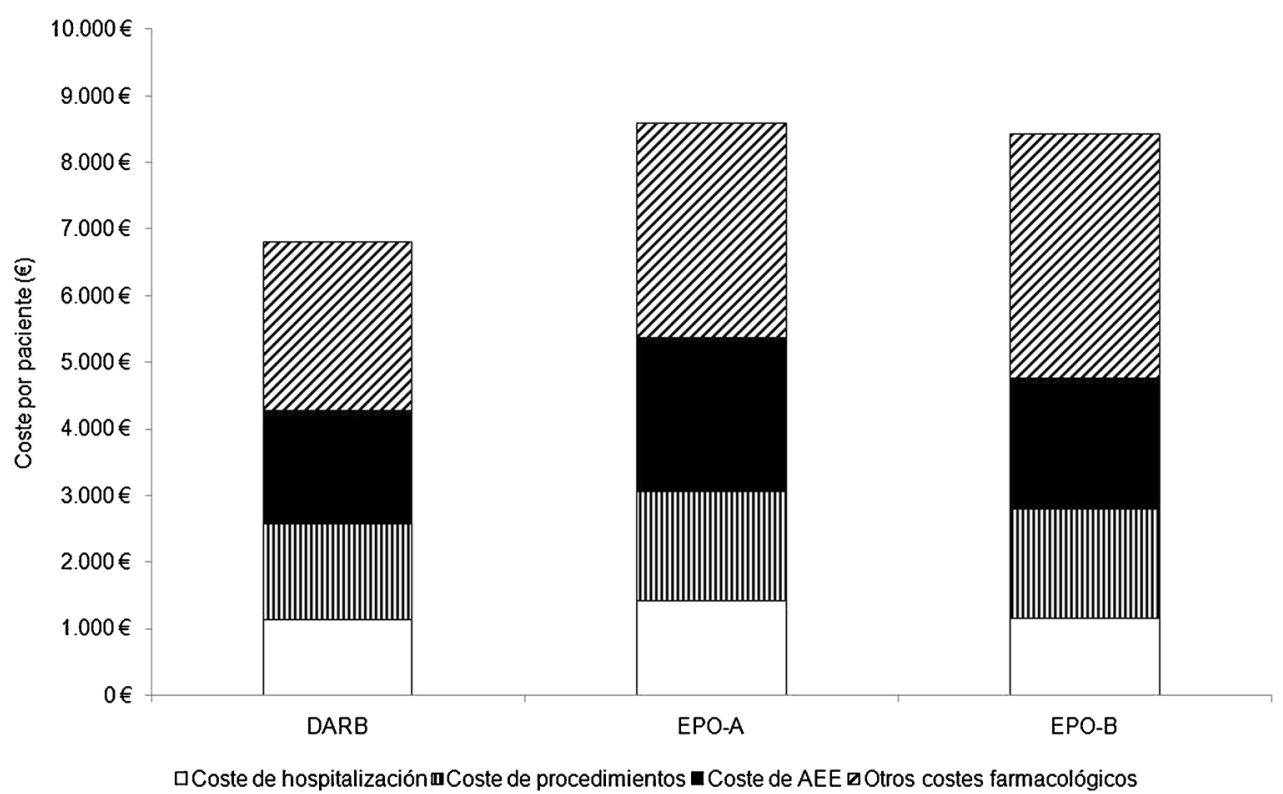

anemia para pacientes tratados con DARB se estimaron en $2.246 €$, mientras que estos mismos costes en pacientes tratados con EPO-A y EPO-B ascendieron a $2.972 €$ $(p<0,0001)$ y $2.425 €(p=0,2531)$, respectivamente. Los costes de los AEE fueron inferiores en los pacientes tratados con DARB en comparación con los tratados con EPO-A (reducción del 26\%; $p<0,0001$ ) o EPO-B (reducción del 13\%; $p=0,0659$ ) (Tabla 5). Las diferencias de costes entre los tres AEE podrían justificarse en base a la menor duración del tratamiento con DARB en comparación con EPO-A y EPO-B. La Fig. 1 y la Fig. 2 presentan de manera desagregada el coste medio total y el coste medio del tratamiento de la anemia en pacientes tratados con AEE. Los resultados del análisis de sensibilidad utilizando el PVP en lugar del PVL, presentados en la Tabla 5, muestran que los costes totales por paciente con DARB fueron de $7.747 €$, un $26 \%$ inferiores a los de EPO-A (10.466 €; $p<0,0001)$ y un $20 \%$ inferiores en comparación con EPO-B (9.723 €; $p=0,0019)$.

\section{Discusión}

Los resultados de este análisis son comparables a los presentados por Spaepen en Bélgica [10], es decir, en España es menos costoso tratar a pacientes con AIQ usando DARB que usando EPO-A o EPO-B, tanto en términos de costes totales de tratamiento (incluyendo los costes de otros tratamientos farmacológicos), como en cuanto a los costes relacionados directamente con el tratamiento de la anemia. Estos resultados son similares a los de otros estudios realizados en España, que concluyeron que el uso de DARB en el tratamiento de la AIQ ofrece una reducción de costes en comparación con el uso de EPO-A o EPO-B [30, 31]. Aunque entre los objetivos de este análisis no se incluía el de 
Figura 2 Coste medio por paciente relacionado con la anemia

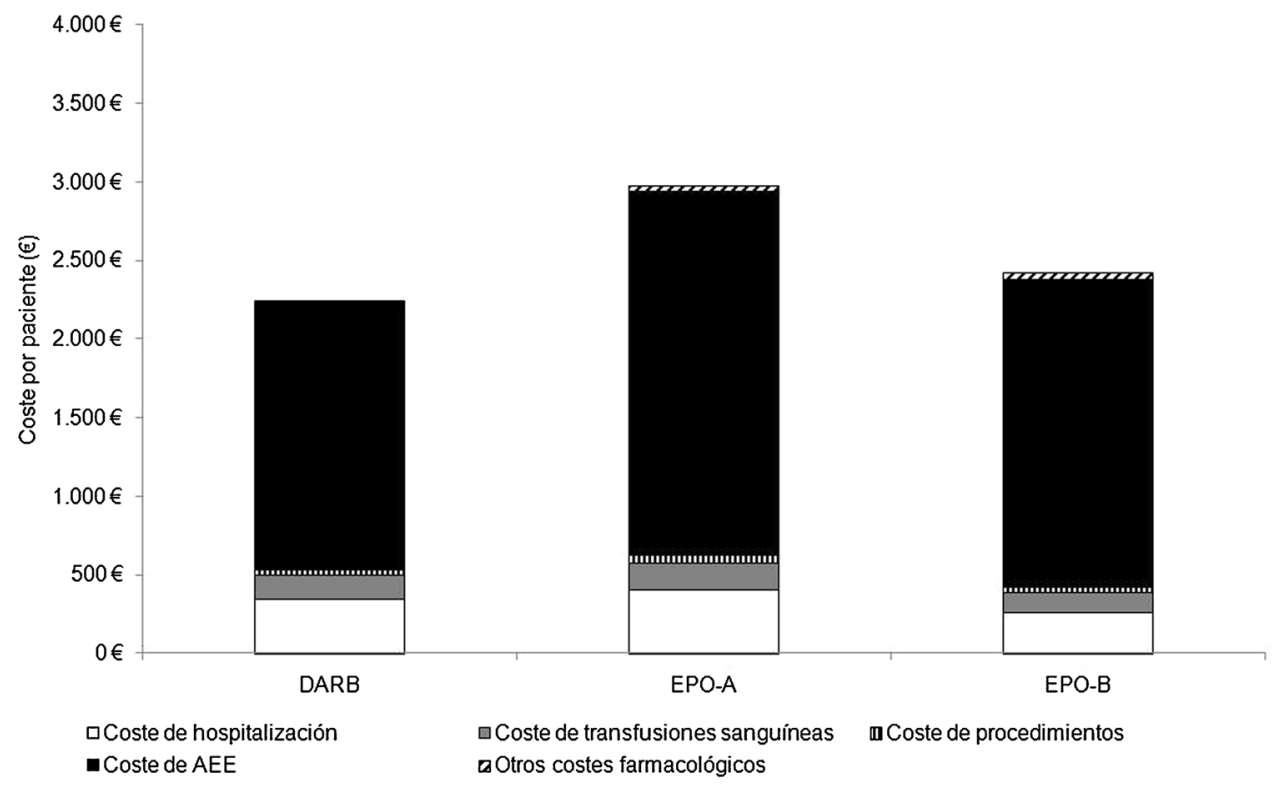

explorar las razones por las que los costes en los pacientes tratados con DARB son más bajos que en los tratados con otros AEE, existen indicios de que la corta duración del tratamiento de DARB puede ser un factor importante [10,32]. El análisis realizado en España encontró diferencias significativas entre las mismas variables que el análisis realizado en Bélgica (por ejemplo, la duración del tratamiento, la duración de la hospitalización y la tasa de hospitalización por la quimioterapia).

Existe evidencia que demuestra que las características clínicas de los pacientes, como por ejemplo el nivel basal de $\mathrm{Hb}$, pueden influir en la elección de los AEE, la dosis utilizada y los costes del tratamiento. En consonancia con las directrices de los estudios de bases de datos retrospectivos de ISPOR [33], Polsky y cols. [34] analizaron la importancia de varios métodos de análisis (como el índice de propensión) para controlar el sesgo de selección de los pacientes cuando se comparan los costes del paciente a nivel individual. El análisis realizado por Polsky y cols. [34] se basó en historias clínicas electrónicas de dos bases de datos de EEUU y comparó el coste del tratamiento de la AIQ con DARB o con EPO-A. El estudio puso de manifiesto la importancia de aparear las características de los pacientes al inicio del estudio, y demostró que el número de parámetros estadísticamente significativos del análisis se redujo en un $83 \%$ cuando se realizaron ajustes en base al nivel basal de $\mathrm{Hb}$. El análisis de Polsky concluyó que el coste del tratamiento con EPO-A era significativamente mayor que el del tratamiento con DARB cuando se realizaron ajustes en las características de referencia [34]. Estos hallazgos destacan la importancia del apareamiento mediante el índice de propensión y son similares a los obtenidos por Spaepen [10], que sirvieron de base para este análisis en España, y demuestran que los costes de tratamiento con DARB son menores que con
EPO-A o EPO-B. Por el contrario, Pashos y cols. [35] publicaron recientemente los resultados de un estudio también basado en historias clínicas electrónicas, en que compararon los beneficios clínicos y económicos del tratamiento de la AIQ con EPO-A y DARB. Los resultados de este análisis, que indican unos costes mayores para los pacientes tratados con DARB, contradicen los obtenidos por Spaepen y cols. [10] y Polsky y cols. [34], lo que puede ser debido a que los autores no realizaron ajustes en las características basales de los pacientes.

Para poder estimar el coste del tratamiento de la AIQ, y utilizar el apareamiento mediante el índice de propensión del análisis original, se hicieron ajustes en el conjunto de datos belga. Se encontraron similitudes tanto en los perfiles demográficos como en los patrones de tratamiento de Bélgica y España. Sin embargo, se incluyeron ajustes en los cuatro tipos de tumores en los que el análisis original encontró diferencias significativas entre los tres grupos de tratamiento, ya que tanto la incidencia del cáncer como el régimen de quimioterapia utilizado influyen en el desarrollo de AIQ y en los costes asociados. Los ajustes realizados en los datos originales sobre el uso de distintos regímenes de quimioterapia y la incidencia de los cuatro tipos de tumores permitieron corregir algunas de las diferencias entre los patrones de tratamiento que pueden existir entre los dos países, lo que reafirma la viabilidad de utilizar los datos ajustados de otro país.

Las bases de datos retrospectivas pueden ofrecer una serie de ventajas sobre los ensayos clínicos cuando se realizan estudios de investigación, como la representatividad de los datos en un entorno real, la reducción de costes y el acceso más rápido a los datos [36]. Sin embargo, entre las limitaciones de este estudio cabe mencionar que el método 
de estandarización utilizado para aplicar las tasas demográficas y epidemiológicas de Bélgica a España no abordaría las diferencias intrínsecas entre los datos epidemiológicos de ambos países. Asimismo, no fue posible tener en cuenta todas las diferencias entre Bélgica y España en cuanto a la gestión de la AIQ, los patrones de tratamiento y los regímenes de quimioterapia usados, lo que puede influir en las diferencias en los costes de gestión y tratamiento. Dado que la elección de los AEE no suele ser determinada por el régimen de quimioterapia utilizado, la sub- o sobre-estimación de los costes debería ser similar para cada uno de los tres AEE; por lo tanto, el impacto en los resultados generales del estudio debería ser mínimo. A pesar de la correlación entre la incidencia de cáncer y la quimioterapia, no sería suficiente ajustar solo en base a la incidencia de cáncer, ya que las discrepancias en las prácticas locales podrían dar lugar a diferencias en las moléculas de quimioterapia utilizadas.

A pesar de los ajustes realizados para tener en cuenta las diferencias de la incidencia del cáncer y el uso de las distintas quimioterapias, la falta de otros datos imposibilita explicar todos los posibles factores de confusión. Algunos de los factores que podrían influir en el resultado final de este análisis incluyen, por ejemplo, la gravedad de la enfermedad, la mortalidad específica de algunas enfermedades o los patrones de uso de hierro intravenoso. Además, las guías actuales sobre el tratamiento de la anemia con DARB también permiten que los pacientes reciban $500 \mathrm{mcg}$ una vez cada tres semanas (Q3W) (no solo los $150 \mathrm{mcg}$ QW disponibles cuando Spaepen y cols. realizaron su estudio) [26]. El hecho de no incluir en este estudio pacientes que recibieran DARB 500 mcg Q3W, se ha considerado un enfoque conservador ya que el tratamiento con DARB $500 \mathrm{mcg}$ Q3W, gracias a la menor frecuencia de administración, probablemente implicaría un mayor ahorro de costes con DARB debido, por ejemplo, a la realización de menos visitas médicas.

En general, la metodología utilizada en este estudio permitió extender el análisis de Bélgica a España. Futuras investigaciones deberían explorar la aplicabilidad de esta metodología a diferentes opciones de tratamiento o procedimientos, tanto en otras áreas de tratamiento/enfermedades distintas como en otros países. Asimismo, los resultados de este análisis deberían confirmarse a través de la realización de un estudio observacional prospectivo llevado a cabo en varios centros en España, con el fin de disponer de datos más reales del coste de los AEE en el tratamiento de la AIQ, en que también se evaluasen las posibles diferencias en los patrones de tratamiento y en los resultados entre diferentes regiones de España.

\section{Conclusión}

Los resultados de este análisis encontraron grandes similitudes entre las poblaciones belgas y española en términos de perfiles demográficos (edad y sexo), a la vez que identificaron diferencias en la incidencia de cuatro tipos específicos de tumores y en los regímenes de quimioterapia. Después de ajustar por las características de referencia de los pacientes, los costes totales asociados al tratamiento con DARB fueron entre un $19 \%$ y un $21 \%$ inferiores a los observados con EPO-B o EPO-A. Los costes relacionados con la anemia fueron entre un $7 \%$ y un $24 \%$ inferiores en los pacientes tratados con DARB, en comparación con los que recibieron EPO-B o EPO-A. Estos resultados son comparables con los del análisis realizado en Bélgica, y demuestran la viabilidad de utilizar esta metodología para adaptar los datos belgas a otros países.

Agradecimientos La financiación de este estudio fue proporcionada por Amgen, S.A.

Open Access This article is distributed under the terms of the Creative Commons Attribution License which permits any use, distribution, and reproduction in any medium, provided the original author(s) and the source are credited.

\section{Bibliografía}

1. Hofman M, Ryan JL, Figueroa-Moseley CD, et al. Cancer-related fatigue: the scale of the problem. Oncologist. 2007;12(Suppl 1):410 .

2. Kimel M, Leidy NK, Mannix S, et al. Does epoetin alfa improve health-related quality of life in chronically ill patients with anemia? Summary of trials of cancer, HIV/AIDS, and chronic kidney disease. Value Health. 2008;11(1):57-75.

3. Cella D, Kallich J, McDermott A, et al. The longitudinal relationship of hemoglobin, fatigue and quality of life in anemic cancer patients: results from five randomized clinical trials. Ann Oncol. 2004;15(6):979-86.

4. Cremieux PY, Finkelstein SN, Berndt ER, et al. Cost effectiveness, quality-adjusted life-years and supportive care. Recombinant human erythropoietin as a treatment of cancer-associated anaemia. Pharmacoeconomics. 1999;16(5 Pt 1):459-72.

5. Rizzo JD, Seidenfeld J, Piper M, et al. Erythropoietin: a paradigm for the development of practice guidelines. Hematology. Am Soc Hematol Educ Program. 2001;1:10-30.

6. Hedenus M, Adriansson M, San MJ, et al. Efficacy and safety of darbepoetin alfa in anaemic patients with lymphoproliferative malignancies: a randomized, double-blind, placebo-controlled study. Br J Haematol. 2003;122(3):394-403.

7. Esquerdo G, Llorca C, Cervera JM, et al. Effectiveness of darbepoetin alfa in a cohort of oncology patients with chemotherapyinduced anaemia. Relationship between variation in three fatiguespecific quality of life questionnaire scores and change in haemoglobin level. Clin Transl Oncol. 2011;13(5):341-7.

8. Khan FA, Shukla AN, Joshi SC. Anaemia and cancer treatment: a conceptual change. Singap Med J. 2008;49(10):759-64.

9. Repetto L. Incidence and clinical impact of chemotherapy induced myelotoxicity in cancer patients: an observational retrospective survey. Crit Rev Oncol Hematol. 2009;72(2):170-9.

10. Spaepen E, Demarteau N, Van Belle S, et al. Health economic evaluation of treating anemia in cancer patients receiving chemotherapy: a study in Belgian hospitals. Oncologist. 2008;13(5):596-607. 
11. Grimes DA, Schulz KF. Bias and causal associations in observational research. Lancet. 2002;359(9302):248-52.

12. Rosenbaum P, Rubin D. The central role of the propensity score in observational studies for causal effects. Biometrika. 1983;70(1):41-55.

13. Rosenbaum P, Rubin D. Reducing bias in observational studies using subclassification on the propensity score. J Am Stat Assoc. 1984;79(387):516-24.

14. Rubin D. Estimating causal effects from large data sets using propensity scores. Ann Intern Med. 1997;127(8 Pt 2):757-63.

15. Abraira V. El control de la confusión en estudios observacionales: el índice de propensión. Semergen. 2003;29(10):529-31.

16. Herrett E, Thomas SL, Schoonen WM, et al. Validation and validity of diagnoses in the general practice research database: a systematic review. Br J Clin Pharmacol. 2010;69(1):4-14.

17. ISPOR International Digest of Databases. International Society for Pharmacoeconomics and Outcomes Research (ISPOR). 2011. Disponible en: http://www.ispor.org/DigestOfIntDB/CountryList. aspx.

18. Kirkwood B, Sterne J. Essential medical statistics. 2nd ed. Oxford: Blackwell Science Ltd.; 2003.

19. portalfarma.com-Organización Farmacéutica Colegial. Consejo General de Colegios Oficiales de Farmacéuticos. 2011. Disponible en: http://www.portalfarma.com/. (Último acceso: octubre de 2010).

20. Registro de Altas de los Hospitales del Sistema Nacional de Salud. Ministerio de Sanidad, Política Social e Igualdad. 2011. Disponible en: http://www.msps.es/estadEstudios/estadisticas/ cmbdhome.htm. (Último acceso: octubre de 2010).

21. Base de Datos de Costes Unitarios eSalud. Oblikue Consulting. 2010. Disponible en: http://www.oblikue.com/.

22. Población total y eventos demográficos (UE-27). Eurostat. 2011. Disponible en: http://appsso.eurostat.ec.europa.eu/nui/show.do? dataset=demo_pjan\&lang=en. (Último acceso: mayo de 2010).

23. Número de tumores invasivos por área, grupo de edad, hombres y mujeres. Registro Belga del Cáncer. 2011. Disponible: http:// www.kankerregister.org/. (Último acceso: mayo de 2011).

24. Conjunto Mínimo Básico de Datos-Hospitalización (CMBDH). Ministerio de Sanidad, Política Social e Igualdad. 2011. Disponible en: http://pestadistico.msc.es/PEMSC25/ArbolNodos. aspx.
25. Clasificación Internacional de Enfermedades, Décima Revisión (CIE-10). National Health Center for Health Statistics. 2011. Disponible en: http://www.cdc.gov/nchs/icd/icd10.htm. (Último acceso: mayo de 2010).

26. Aapro M, Link H. September 2007 update on EORTC guidelines and anemia management with erythropoiesis-stimulating agents. Oncologist. 2008;13(Suppl 3):33-6.

27. IMS Health. IMS Multinational Integrated Data Analysis System (MIDAS) Quantum. 2011. IMS Health.

28. ATC Guidelines 2010. European Pharmaceutical Market Research Association. 2011. Disponible en: http://www.ephmra.org/PDF/ ATC\%20Guidelines\%202010.pdf.

29. Duran A, Spaepen E, Lamotte M, et al. Cost analysis: treatment of chemotherapy-induced anemia with erythropoiesis-stimulating agents in five European countries. J Med Econ. 2012;15(3):1-10.

30. Pérez-Alcántara F, Badía Llach X, Vieta Salomó A, et al. Modelo de impacto presupuestario de la administración de darbepoetina alfa cada 3 semanas comparado con la administración de epoetina alfa y epoetina beta 3 veces por semana y semanalmente en pacientes con anemia inducida por quimioterapia. Rev Esp Econ Salud. 2008;7(1):41-8.

31. Sanz Granda A. Análisis de costes del tratamiento de la anemia inducida por la quimioterapia. Evaluación de darbepoetina alfa versus epoetina alfa. Rev Esp Econ Salud. 2009;8(2):93-9.

32. Song X, Long SR, Marder WD, et al. The impact of methodological approach on cost findings in comparison of epoetin alfa with darbepoetin alfa. Ann Pharmacother. 2009;43(7):1203-10.

33. Motheral B, Brooks J, Clark MA, et al. A checklist for retrospective database studies-report of the ISPOR task force on retrospective databases. Value Health. 2003;6(2):90-7.

34. Polsky D, Eremina D, Hess G, et al. The importance of clinical variables in comparative analyses using propensity-score matching: the case of ESA costs for the treatment of chemotherapyinduced anaemia. Pharmacoeconomics. 2009;27(9):755-65.

35. Pashos CL, Larholt K, Fraser KA, et al. Outcomes of erythropoiesis-stimulating agents in cancer patients with chemotherapy-induced anemia. Support Cancer Care. 2011;20(1): 159-65.

36. Sax MJ. Essential steps and practical applications for database studies. J Manag Care Pharm. 2005;11(1 Suppl A):S5-S8. 